\title{
Erratum to: Synergistic anti-malarial action of cryptolepine and artemisinins
}

\author{
Arnold D. Forkuo ${ }^{1}$, Charles Ansah ${ }^{1 *}$, Kwesi M. Boadu', Johnson N. Boampong², Elvis O. Ameyaw², Ben A. Gyan², \\ Andrea T. Arku $^{3}$ and Michael F. Ofori ${ }^{3}$
}

\section{Erratum to: Malar J (2016) 15:89 DOI 10.1186/s12936-016-1137-5}

After publication of the original article [1], the authors found that there was an error in the first sentence of the 'Conclusions' section: "The combination of CPE with the artemisinins showed a synergistic effect both in vivo and in vitro against $P$. falciparum 3D7 and $P$. berghei NK-65, respectively."

Within this sentence, the appearance of ' $P$. falciparum 3D7' and 'P. berghei NK-65' should have been reversed. The sentence in the original article has been corrected to read as follows: "The combination of CPE with the artemisinins showed a synergistic effect both in vivo and in vitro against $P$. berghei NK-65 and P. falciparum 3D7, respectively."

\begin{abstract}
Author details
${ }^{1}$ Department of Pharmacology, Faculty of Pharmacy and Pharmaceutical Sciences, College of Health Sciences, Kwame Nkrumah University of Science and Technology, Kumasi, Ghana. ${ }^{2}$ Department of Biomedical and Forensic Sciences, School of Biological Science, College of Agriculture and Natural Sciences, University of Cape Coast, Cape Coast, Ghana. ${ }^{3}$ Department of Immunology, Noguchi Memorial Institute for Biomedical Research, University of Ghana, Legon, Ghana.
\end{abstract}

The online version of the original article can be found under doi:10.1186/s12936-016-1137-5.

Published online: 16 March 2016

\author{
Reference \\ 1. Forkuo AD, Ansah C, Boadu KM, Boampong JN, Ameyaw EO, Gyan \\ BA, Arku AT, Ofori MF. Synergistic anti-malarial action of cryptolepine \\ and artemisinins. Malar J. 2016;15:89.
}

\footnotetext{
*Correspondence: cansah.pharm@knust.edu.gh

${ }^{1}$ Department of Pharmacology, Faculty of Pharmacy and Pharmaceutical Sciences, College of Health Sciences, Kwame Nkrumah University

of Science and Technology, Kumasi, Ghana
} 